\title{
Quality of Life in Patients with Systemic Lupus \\ Erythematosus
}

\author{
Aya M. Ahmed ${ }^{1}$, Mohammed A. Ismail ${ }^{2}$,SaharA. Elsayed ${ }^{3}$ and Rabab H. \\ $\mathrm{Ali}^{4}$
}

Department of Rheumatology and Rehabilitation ${ }^{1,2,3,4}$, Faculty of Medicine, Sohag University

\section{Abstract}

Introduction: Systemic lupus erythematosus (SLE) is a chronic disease that results in variable and occasionally life-threatening manifestations. persons with SLE may experience a wide range of physical, psychological and social problems that affect quality of life.

Aim of the work: Assess quality of life in the patients suffering from systemic lupus erythromatosus.

Patients and Methods: The study was conducted on forty patients, with systemic lupus erythematosus [32 females ( $80 \%$ ) and 8 males (20\%)] and forty healthy adults of matched age and sex, as control group [30 female (80\%) and 10 males (20\%)]. The patients selected from the rheumatology outpatient clinic of Sohag University hospital. An informed written consent had been taken from all patients.

Results: The increase in the disease activity, as assessed bysystemic lupus erythematosus disease activity index (SLEDAI) was significantly associated with more impaired quality of life as assessed by SF-36 and systemic lupus erythematosus quality of life questionnaire (SLEQOL).

Conclusion:SLE patients have more significantly impaired quality of life, as assessed by short form-36(SF-36), and systemic lupus erythematosus quality of life questionnaire (SLEQOL) than the controls.

Key words: SLE, Quality of life.

\section{Introduction}

The quality of life can be defined as "an individual's perception of their position in life in the context of the culture and value systems in which they live and in relation to their goals, expectations, standards and concerns". This concept includes the person's physical health, psychological state, level of independence, social relationships, personal beliefs and relationship to salient features of surrounding environment. The majority of chronic diseases hold the potential to worsen the overall health of patients by limiting their capacity to live well, limit the functional status, productivity and Health Related Quality of Life (HRQOL) and are a major contributor to health care costs ${ }^{(\mathbf{1})}$.

One of these chronic diseases is systemic lupus erythematosus (SLE), a disorder in which the immune system attacks the tissues and organs of the body, causing inflammation and damage. This most commonly affects women of childbearing age, but also children, adolescents and men, with a prevalence ranging from 20 to 150 cases per 100,000 population, and the 10 -year survival rate is about $70 \%{ }^{(2)}$.

SLE is characterized by relapses and remissions. The new treatment strategies developed for lupus over recent decades have improved survival of patients significantly, and the assessment of health-related quality of life (HRQOL) has became an important outcome measure for patients with lupus ${ }^{(2)}$.

Regarding the areas of quality of life lupus affects alertness, recreation, leisure, sleep, rest, housework, social interaction, communication, mobility, 
the ability to work and emotional balance $^{(2)}$.

Measurement of HRQOL has traditionally relied on two basic approaches: the use of generic questionnaires and the use of diseasespecific questionnaires. Generic questionnaires were developed for general use and may be used in a variety of diseases and population. They allow for comparison with other groups and other conditions and allow measurement of dysfunction for individuals experiencing more than one condition. In contrast, disease-specific questionnaires are designed to measure outcomes in a specific disease. Because they incorporate elements specific to particular diseases, they are believed to be more responsive than generic instruments ${ }^{(2)}$.

At present, the most commonly used measure of HRQOL is the SF-36. Developed by Ware et al. ${ }^{(3)}$, the SF36 is a generic, 36-item self-report questionnaire designed to be used in a variety of conditions, populations, and settings. It includes eight subscales (physical functioning, social functioning, role limitations due to physical problems, role limitations due to emotional problems, mental health, energy/vitality, pain and general health perception) that can be summarized into two component scores: the physical component summary score and the mental component summary score. The SF-36 has been shown to be a valid and reliable instrument in SLE (3)

\section{Aim of the work:}

This study aimed to assess quality of life in the patients suffering from systemic lupus erythematosus.

\section{Patients and Methods:}

Design:Case control study.

Patients:

Our study started from December 2016

to December 2017. It was conducted on forty patients, with systemic lupus erythematosus [32 females (80\%) and 8 males (20\%)] and forty healthy volunteers of matched age and sex, as control group [30 female (80\%) and 10 males $(20 \%)]$.

The patients selected from the rheumatology outpatient clinic of Sohag University hospital. An informed written consent had been taken from all patients. All patients diagnosed according to the Systemic Lupus International Collaborating Clinics (SLICC) Classification Criteria for Systemic Lupus Erythematosus. The patient was classified as having SLE when he or she satisfied 4 of the clinical and immunologic criteria used in the SLICC classification criteria, including at least one clinical criterion and one immunologic criterion, OR if he or she had biopsy-proven nephritis compatible with SLE in the presence of ANAs or anti-dsDNA antibodies. Most of the patients were on treatment in the form of azathioprine, hydroxychloroquine, renoprotective agents as angiotensin converting enzyme inhibitor, low dose steroid, calcium and vitamin D.

\section{Methods:}

The following details obtained from the patients:-

- Demographic data

- age

- sex

- Marital status

- Occupation

- Clinical information

- Duration of SLE (The disease duration had been calculated from the time when the patients' SLE was first diagnosed to the enrollment of the patients to the study)

- Medical and rheumatological history taking with a special focus on symptoms of SLE

- Careful general, musculoskeletal examination.

- Laboratory tests include

- complete blood count

- creatinine 
- liver enzymes

- erythrocyte sedimentation rate (ESR)

- complete urine analysis

- anti-nuclear antibody (ANA)

- anti-double-stranded DNA antibody (anti-ds-DNA)

- 24 hour urinary protein

Exclusion criteria:

- Any patient with any collagen disease mimic SLE.

- Any patient clinically diagnosed as neuropsychiatric lupus.

Assessment of the Disease activity in the patients was done using the SLE Disease Activity Index (SLEDAI):

- The systemic lupus erythrematosus disease activity index (SLEDAI) is a scale specific to assess disease activity in adults with SLE. It measures potentially reversible manifestations of the underlying inflammatory disease process. The scale consists of 24 "weighted" attribute grouped into 9 domains (organ systems) The final score comprises the sum of all weighted attributed scores (Bombardier et al., 1992).

\section{Grading of disease activity:}

- Mild activity: 1-10

- Moderate activity: 11-20

- Severe activity: 21-45

- Very severe activity: $>45$

Assessment of the quality of life in the patients and the control group using SF-36 questionnaire:

- $\quad$ The short form 36 (SF-36) questionnaire is a generic instrument used to assess the quality of life. The scores are based on responses to individual questions, which are summarized into eight scales, each of which measures a health concept. These scales include function domains and aspects of well-being, as follows: physical function (PF-10 questions) (limitations in physical activities because of health problems); rolephysical (RP-4 questions) (limitations in usual role activities because of physical health problems); bodily pain (BP- 2 questions) (influence of pain on daily activities); vitality (VT- 4 questions) (energy level and fatigue); role-emotional problems; mental health (MH-3 questions) (psychological distress and well-being); social function (SF-2 questions), (limitations in social activities because of physical or emotional problems) ; general health (GH- 6 questions) (subjective perception of health status). These domains represented by 36 questions (items), the patients in our study were asked to answer these questions. sum of each item score for total score with higher scores reflect better health.

- All questions were scored on a scale from 0 to 100 , with 100 representing the highest level of functioning possible. Aggregate scores were compiled as a percentage of the total points possible, using the scoring table (STEP I chart). The scores from those questions that address each specific area of functional health status (STEP II chart) were then averaged together, for a final score within each of the 8 dimensions measured. (eg pain, physical functioning etc.).

- For example, to measure the patients energy/fatigue level, we added the scores from questions 23, 27, 29, and 31 . If a patient circled 4 on 23, 3 on 27, 3 on 29 and 1on 31. An answer of 4 to Q23 was scored as 40, 3 to Q27 was scored as 60, 3 to Q29 was scored as 40 and 1 to Q31 was scored as 0 . The score for this block was $40+60+40$ $=140$. Now we divided by the 4 answered questions to get a total of 46.7. Since a score of 100 represents high energy with no fatigue, the lower score of $46.7 \%$ suggests the patient is experiencing a loss of energy and is experiencing some fatigue. 


\section{Results}

The age of our patients ranged from 18 to 54 years, the mean was $31 \pm 9.13$ years. The mean duration of disease was $3 \pm 3.8$ years, and it ranges from 1 to 15 years. 17 patients have disease duration of one year or less. 5 patients have disease duration of 1-2 years.8 patients have disease duration of 2-5 years and 10 patients have disease duration of more than 5 years. 24 of our patients had mild disease activity and 16 had moderate to sever disease activity according to SLEDAI score. Significant $(p<0.001)$ difference in the physical function between cases and controls. Also there was significant $(\mathrm{p}<0.001)$ difference in the body pain, general health, social function, role-emotional, mental health and vitality between the cases and controls. Also there was significant $(p<0.001)$ difference of total SF-36 between the cases and controls. There was a negative and highly significant $(p<0.001)$ correlation between SLEDAI and SF36. There was negative relation between total SF-36 score and disease activity assessed by SLEDAI. When we made correlation between SLEQOL and disease activity assessed by SLEDAI, we found that there was moderate positive and significant $(p=0.015)$ correlation between SLEDAI and SLEQOL. There is a significant difference $(P=0.002)$ in total SF-36 score between mild disease activity group and moderate to severe disease activity group. There is a higher total SF-36 score in mild disease activity group than in moderate to severe disease activity group. There was a significant difference $(P=0.006)$ in SLEQOL score between mild disease activity group and moderate to severe disease activity group as shown in table 1. There is a lower SLEQOL score in mild disease activity group than that of moderate to severe

disease activity

group.

Table 1 show comparison of SLEQOL score in mild and moderate to severe disease activity groups of patients

\begin{tabular}{lll}
\hline Group & Mean \pm SD & P value \\
\hline Mild disease & $83.167 \pm$ & $\mathbf{0 . 0 0 6}$ \\
activity & 24.347 & \\
\hline $\begin{array}{l}\text { Moderate to severe } \\
\text { disease activity }\end{array}$ & $107.625 \pm 28.362$ & \\
\hline
\end{tabular}

There was negligible and non-significant $(p=0.8 / p=0.6)$ correlation between both SF36 and SLEQOL to duration of disease.There was a negative and nonsignificant $(p=0.09)$ correlation between age of patients and SF36, but there was a weak positive and non-significant $(p=0.39)$ correlation between SLEQOL and age of patients

as shown

in

table

2.

Table 2. Correlation of SF-36 and SLEQOL to age of the patients

\begin{tabular}{lll} 
Age of the patients & SLEQOL & SF36 \\
$r$ & 0.139 & $\mathbf{- 0 . 2 6 5}$ \\
$P$ & 0.393 & 0.099 \\
\hline
\end{tabular}

\section{Discussion}

According to our results all domains of sf-36 (physical functioning rolephysical, bodily pain, general health, vitality, social functioning, roleemotional, and mental health) were higher in controls when compared with patients and the difference between the two groups was statistically significant.

Also the total SF-36 score was higher in controls than patients with statistically significant difference between the two groups, which is consistant with impaired QOL in lupus patients.

Our result are in agreement with Alarcón et al. ${ }^{(4)}$ who found that the 
scores for the eight subscales of the SF-36 were also lower in the patients than those for the general population. Near to our results, Rinaldi et al. ${ }^{(5)}$ found that in all domains the mean scores were lower in SLE patients than in controls. The differences were statistically significant for all domains except role-physical and social functioning.

Our results are near to the results of Yilmaz-Oner et al. ${ }^{(6)}$ as they found that all domains of SF-36 questionnaires were low lupus patients and consistent with impaired QOL,and was in line with Barnado et al. ${ }^{(7)}$ who reported that the way patients perceive the impact of disease on their physical, emotional, and social function, or health-related quality of life (HRQOL), is poor in patients with systemic lupus erythematosus (SLE) compared with the general population, regardless of the measure used.

Our results were also in agreement with MoK et al. ${ }^{(8)}$ who demonstrated that impaired HRQOL is more common in SLE patients than controls, regardless of age, sex, education and poverty.

Regarding the relation between disease activity and quality of life assessed by SF-36 in our study, we found that there was negative and highly significant correlation between SLEDAI and SF36 , total scores of SF-36 decrease with the increase in SLEDAI score. Also there was a significant difference in total SF-36 score between mild disease activity group and moderate tosevere disease activity group. There was a higher total SF-36 score in mild disease activity group than in moderate to severe disease activity group. This means that increased disease activity is associated with impaired QOL, this was close to the results of Chaigne et al. ${ }^{(9)}$ which showed a reduction in most HRQOL outcomes in patients with active SLE, when assessed by the SF36.

Also Shen et al. ${ }^{(10)}$ showed that the total SF-36 score was affected by disease activity in both direct and indirect ways.Our results are in accordance with Khanna et al. ${ }^{(11)}$ who reported that SF-36 scores were negatively correlated with BILAG and SLEDAI scores.

On the other hand, regarding the relation between disease activity and quality of life assessed by SLEQOL in our study, we found moderate positive and significant correlation between SLEDAI and SLEQOL, SLEQOL scores decrease with the increase in SLEDAI score. Also there was a significant difference in SLEQOL score between mild disease activity group and moderate to severe disease activity group. There was a lower SLEQOL score in mild disease activity group than that of moderate to severe disease activity group. This means that lower disease activity is associated with less impaired QOL.

Our results are in accordance with Freire et al. ${ }^{(12)}$ that showed that SLEQOL have moderate correlation with disease activity measured using SLEDAI, On the other hand the results of Leong et al. ${ }^{(13)}$ showed poor correlation between SLEQOL and SLEDAI, also Kasitanon et al. ${ }^{(14)}$ found that the Thai version of the SLEQOL correlated weakly with the SLEDAI.

In our study, we found negative and non significant correlation between age of the patients and SF36, and weak and non significant correlation between SLEQOL and age of patients, so the increase in age was associated with decrease in total SF-36 score and increase in SLEQOL score, and both of them are consistant with impaired quality of life. This was in agreement with results of Yilmaz-Oner et al. ${ }^{(6)}$ as they found negative and non 
significant correlation between SF-36 score and the age of patients. Rinaldi et al. ${ }^{(15)}$ also reported that in Italian people with SLE, SF-36 score tended to decrease with age but the relation was significant. Regarding the duration of the disease, there was negligible and non significant correlation between both SF36, SLEQOL and duration of disease that means no association between HRQOL and disease duration. This was in agreement with studies ofJolly et al ${ }^{(16)}$, Khanna et al. ${ }^{(11)}$, all of themreportedthat disease duration did not affect HRQOL.

\section{Conclusion:}

- SLE patients have more significantly impaired quality of life, as assessed by SF-36, and SLEQOL than the controls.

- The increase in the disease activity, as assessed by SLEDAI was significantly associated with more impaired quality of life as assessed by SF-36 and SLEQOL.

- There was no relation between the impaired quality of life in lupus patients and the disease duration.

\section{Recommendations}

We recommend that:

- Use of SF-36 questionnaire and SLEQOL questionnaire in clinical trials studies of SLE patients to evaluate the burden of all aspects of SLE on patients' life as a chronic disease.

- Assessment of health-related quality of life (HRQOL) alongside measure of disease activity provides a more comprehensive and holistic picture of the patient and their disease.

\section{References:}

1. Megari K. Quality of Life in Chronic Disease Patients. Health psychology research. 2013;1(3):e27.

2. Tsokos GC. Systemic lupus erythematosus. The New England journal of medicine. 2011;365(22):2110-21.
3. Ware JE, Jr., Sherbourne CD. The MOS 36-item short-form health survey (SF-36). I. Conceptual framework and item selection. Medical care. 1992;30(6):473-83.

4. Alarcon GS, McGwin G, Jr., Uribe A, Friedman AW, Roseman JM, Fessler BJ, et al. Systemic lupus erythematosus in a multiethnic lupus cohort (LUMINA). XVII. Predictors of selfreported health-related quality of life early in the disease course. Arthritis and rheumatism. 2004;51(3):465-74.

5. Doria A, Rinaldi S, Ermani M, Salaffi F, Iaccarino L, Ghirardello A, et al. Health-related quality of life in Italian patients with systemic lupus erythematosus. II. Role of clinical, immunological and psychological determinants. Rheumatology. 2004;43(12):1580-6.

6. Yilmaz-Oner S, Oner C, Dogukan FM, Moses TF, Demir K, Tekayev N, et al. Health-related quality of life assessed by LupusQoL questionnaire and SF-36 in Turkish patients with systemic lupus erythematosus. Clinical rheumatology. 2016;35(3):617-22.

7. Barnado A, Wheless L, Meyer AK, Gilkeson GS, Kamen DL. Quality of life in patients with systemic lupuserythematosus (SLE) compared with related controls within a unique African American population. Lupus. 2012;21(5):563-9.

8. Mok CC, Ho LY, Cheung MY, Yu KL, To $\mathrm{CH}$. Effect of disease activity and damage on quality of life in patients with systemic lupus erythematosus: a 2-year prospective study. Scandinavian journal of rheumatology. 2009;38(2):121-7.

9. Chaigne B, Chizzolini C, Perneger T, Trendelenburg M, Huynh-Do U, Dayer $\mathrm{E}$, et al. Impact of disease activity on health-related quality of life in systemiclupus erythematosus - a crosssectional analysis of the Swiss Systemic Lupus Erythematosus Cohort Study (SSCS). BMC immunology. 2017;18(1):17. 
10. Shen B, Feng G, Tang W, Huang X, Yan $\mathrm{H}, \mathrm{He}$ Y, et al. The quality of life in Chinese patients with systemic lupus erythematosus is associated with disease activity and psychiatric disorders: a path analysis. Clinical and experimental rheumatology. 2014;32(1):101-7.

11.Khanna S, Pal H, Pandey RM, Handa $R$. The relationship between disease activity and quality oflife in systemic lupus erythematosus. Rheumatology. 2004;43(12):1536-40.

12. Freire EA, Bruscato A, Leite DR, Sousa TT, Ciconelli RM. Translation into Brazilian Portuguese, cultural adaptation and validatation of the systemic lupus erythematosus quality of life questionnaire (SLEQOL). Acta reumatologica portuguesa. 2010;35(3):334-9.

13.Leong KP, Kong KO, Thong BY, Koh ET, Lian TY, Teh CL, et al. Development and preliminary validation of a systemic lupus erythematosus-specific quality-of-life instrument) SLEQOL). Rheumatology. 2005;44(10):1267-76.

14. Kasitanon N, Wangkaew S, Puntana S, Sukitawut W, Leong KP, Tan Tock Seng Hospital Lupus Study G, et al. The reliability, validity and responsiveness of the Thai version of Systemic Lupus Erythematosus Quality of Life (SLEQOL-TH) instrument. Lupus. 2013;22(3):289-96.

15.Rinaldi S, Doria A, Salaffi F, Ermani M, Iaccarino L, Ghirardello A, et al. Health-related quality of life in Italian patients with systemic lupus erythematosus. I. Relationship between physical and mental dimension and impact of age. Rheumatology. 2004;43(12):1574-9.

16.Jolly M, Pickard SA, Mikolaitis RA, Rodby RA, Sequeira W, Block JA. LupusQoL-US benchmarks for US patients with systemic lupus erythematosus. The Journal of rheumatology. 2010, 37:1828-33. 